\title{
Hypertrophic Cardiomyopathy and Tricuspid Regurgitation with Right Heart Failure Mimicking Constrictive Pericarditis: A Rare Case Report
}

\author{
SMG SAKLAYEN ${ }^{1}$, RAKIBUL HASAN ${ }^{1}$, REDOY RANJAN ${ }^{1}$, MOSTAFIZUR RAHMAN ${ }^{1}$, \\ REZWANUL HOQUE ${ }^{1}$, ASIT BARAN ADHIKARY ${ }^{1}$ \\ ${ }^{1}$ Department of Cardiac Surgery, Bangabandhu Sheikh Mujib Medical University, Dhaka \\ Address for corrospondance: Dr SMG Saklayen, Medical Officer, Department of Cardiac Surgery, Bangabandhu Sheikh Mujib \\ Medical University, Dhaka. E mail: saklayendmc@gmail.com
}

\begin{abstract}
:
Cardiomyopathy is the measurable deterioration of the function of the myocardium for any reason, usually leading to heart failure. Tricuspid regurgitation may result from structural alterations of any one or all of the components of the tricuspid valve apparatus which include the leaflets, chordae tendinae, annulus, and papillary muscles or adjacent right ventricular muscle. We are reporting a case of Cardiomyopathy with tricuspid regurgitation with right heart failure in a 38 years male. Preoperatively he was diagnosed as a case of constrictive pericarditis. Diagnosis of Tricuspid regurgitation with cardiomegaly was confirmed peroperatively. During operatrion tricuspid valve anatomy dimunited and severe tricuspid regurgitation was identified. Grossly dilated RA, RV identified and other anatomy of heart was normal. De-Vega Tricuspid anuloplasty done with pledgeted stich 2-0 polyster. Part of right atrial wall (2X2.5 inch) excised and resected portion sent for histopathology. Postoperative course was uneventful with marked improvement of symptoms.
\end{abstract}

\section{Introduction}

Hypertrophic cardiomyopathy (HCMP) is a heart disease instigated by a variety of causes, such as genetic syndromes, e.g., Noonan syndrome and Costello syndrome, metabolic diseases, such as long-chain 3hydroxyacyl-CoA dehydrogenase deficiency and adenosine triphosphate (ATP) synthase deficiency, familial history, maternal diabetes mellitus, steroid use ${ }^{1}$. Hypertrophic cardiomyopathy (HCM) is a disease of the myocardium with autosomal dominant pattern of inheritance $^{2}$. It is characterized by inappropriate hypertrophy involving either the interventricular septum, apex or left ventricular free wall in isolation or combined ${ }^{2}$. Secondary tricuspid dilatation may or may not be accompanied by tricuspid regurgitation (TR). Tricuspid dilatation can be objectively measured whereas TR can vary according to the preload, afterload, and right ventricular function ${ }^{3}$. Tricuspid regurgitation is caused by structural or functional aetiologies ${ }^{4}$. Structural tricuspid insufficiency results from either congenital heart disease or from acquired pathology, which includes rheumatic heart disease endocarditis, blunt trauma with rupture of a papillary muscle or chordae tendineae, carcinoid disease, myxomatous degeneration, and collagen-vascular disease ${ }^{6}$.
In case of right heart failure physical examination may reveal pitting peripheral edema, ascites, and hepatomegaly. Jugular venous pressure is frequently assessed as a marker of fluid status, which can be accentuated by eliciting hepatojugular reflux. If the right ventricular pressure is increased, a parasternal heave may be present, signifying the compensatory increase in contraction strength. Backward failure of the right ventricle leads to congestion of systemic capillaries. This generates excess fluid accumulation in the body. This causes swelling under the skin and usually affects the dependent parts of the body first. Nocturia may occur when fluid from the legs is returned to the bloodstream while lying down at night. In progressively severe cases, ascites and hepatomegaly may develop. Significant liver congestion may result in impaired liver function, and jaundice and even coagulopathy may occur $^{6}$.

\section{Case report:}

A 38 years old male presented with repeated attack of swelling of abdomen and both legs, respiratory difficulty for 3 years. The general examination revealed no anaemia, cyanosis, jaundice, clubbing dehydration but there was leg edema, ascites with raised JVP. Cardiovascular 
examination showed S1 and S2 soft and muffled and $3^{\text {rd }}$ heart sound present. Patients Hb\% 12.3 g/dl, ESR was 25 $\mathrm{mm}$ in $1^{\text {st }}$ hour, total W.B.C. count was $8000 / \mathrm{cmm}$, Neutrophil 74\%, Lymphocyte $17 \%$, Monocyte 06\%, Serum Albumin $37 \mathrm{gm} / \mathrm{L}$. Chest X ray showed heart enlarge in TD with globular shaped heart (Fig-1), ECG reveals RBBB spirometry moderate restriction. 2D and M mode and colour Doppler echocardiography showed pericardium is thickened, LA, LV dilated, exaggerated inspiratory variation in tricuspid and mitral inflow, Trivial MR, Grade II TR, Dilated IVC with reduce collapsing. All the features are consistent with constrictive pericarditis with Good LV systolic function. Ultrasonography of the whole abdomen showed congestive hepatomegaly with marked ascites. The patient underwent operation with an intention to do pericardiectomy. With all asceptic precaustion standard median sternotomy done under general anaesthesia. Our initial plan was to do pericardiactomy but peroperatively we found pericardium absolutely normal, minimal pericardial collection with hugely dilated right atrium. As no need of doing pericardiactomy, in this scenario we plan to establish cardiopulmonary bypass (CPB) to arrest the heart. Right atriotomy done, atrial wall found hypertrophied, severe tricuspid regurgitation was identified, other structures reveals normal finding. Tricuspid annuloplasty done using De Vega procedure, part of right atrial wall (2X2.5 inch) excised (Fig-2). Right atrium closed in layer. Patient was gradually weaned from cardiopulmonary bypass smoothly with minimum inotropic

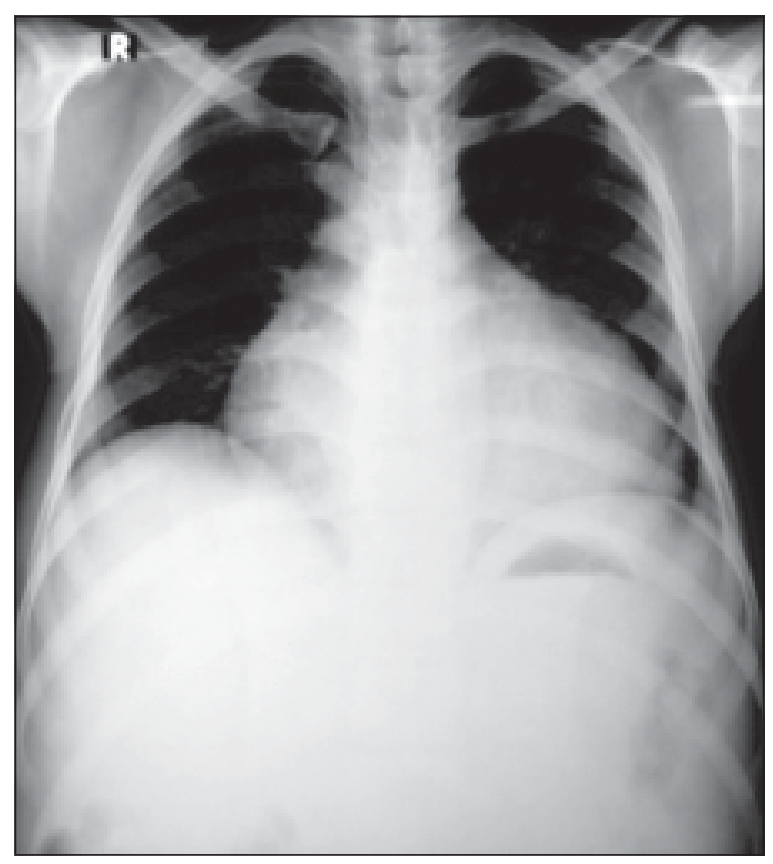

Fig.-1: Chest Xray PA view showing cardiomegaly support. Chest wall was closed in layer. Atrial wall was sent for histopathology and report showed hypertrophied cardiomyopathy (Fig-3). Post-operative recovery was uneventful. Patient was doing well and performing his daily activities with minimum difficulty. So he was discharged on $19^{\text {th }}$ POD with advice to follow up after 3 weeks.

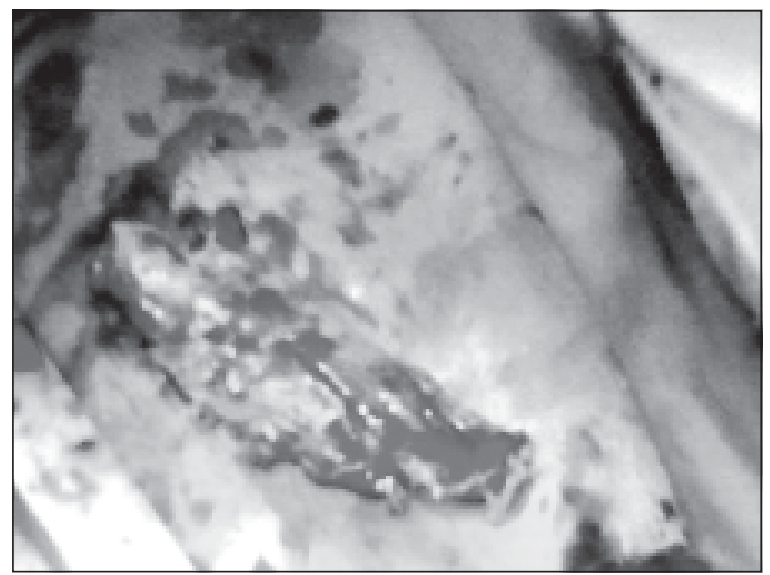

Fig.-2: Resected part of right atrium (2X2.5 inches)

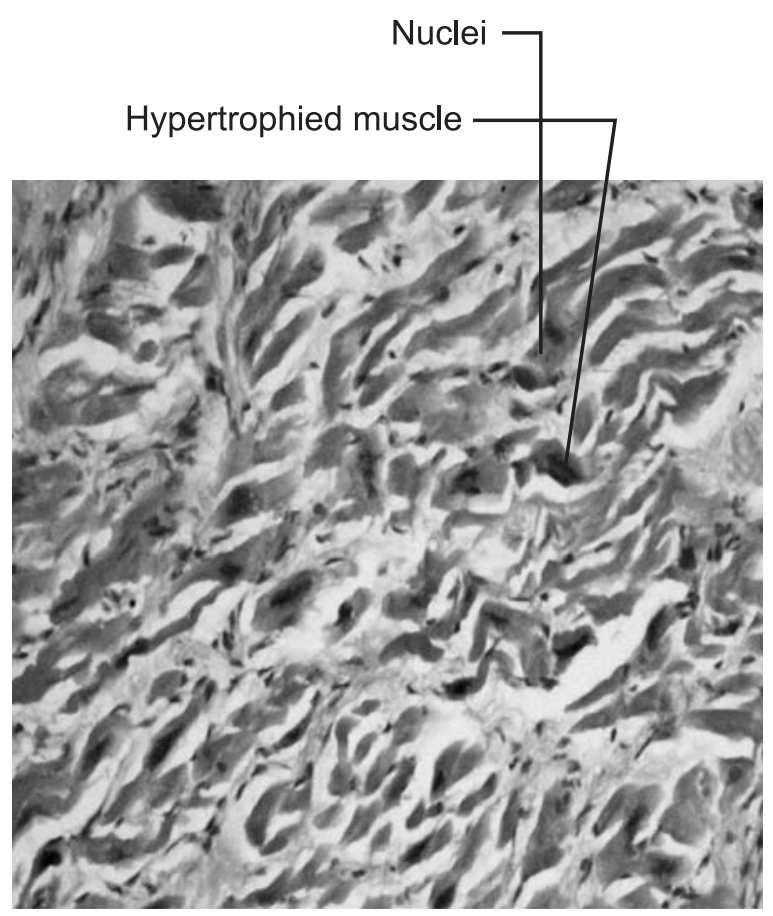

Fig.-3: Photomicrograph (220X magnification) of RA wall with Haematoxylin and Eosin stain showing hypertrophy of cardiac muscle with fibrosis of surrounding tissue (Courtesy: Department of Pathology, BSMMU)

\section{Discussion:}

This patient was initially referred by cardiologist with the diagnosis of constrictive pericarditis and echocardiographic report was in favour of constrictive 
pericarditis. But peroperatively as the pericardium was found normal and as there was huge cardiomegally we planned to arrest the heart with the help of cardiopulmonary bypass to find out the cause of cardiomegally. We found severe tricuspid regurgitation with hugely dilated right atrium. So we had done DeVega annuloplasty of tricuspid valve with right atrioplasty. No case was reported having same scenario, that is, tricuspid regurgitation with cardiomyopathy with right heart failure. But several cases were reported having either tricuspid regurgitation or hypertropnic cardiomyopathy. Hyeon Seok Seo and his colleague worked on a Case of Congenital Hypertrophic Cardiomyopathy ${ }^{1}$. They considered performing a septal myectomy but the patient's myocardium showed aggravation with rapid thickening and he ultimately died from congestive heart failure and respiratory failure, due to pulmonary congestion ${ }^{1}$. We had done right atrioplasty by removing part of right atrial wall. Post operatively his sign symptoms of right heart failure improved. Ram Sharony and colleague managed several case of tricuspid regurgitation and they proved that prosthetic replacement of the tricuspid valve has produced disappointing results, with mortality up to 36 percent $^{5}$. Therefore, they prefer posterior annuloplasty and their experience shown that with more than 300 patients from their institution shows that the posterior leaflet annuloplasty is simple, safe, and reproducible in the absence of significant intrinsic leaflet disease $^{5}$. Jason H. Rogers and his colleagues worked on evolving management of tricuspid regurgitation and they came to the conclusion that DeVega annuloplasty appear to have a lower freedom from recurrent TR than rigid annuloplasty rings ${ }^{6}$. To correct tricuspid regurgitation we follow the posterior annuloplasty of DeVega style and postoperatively patient was doing fine with clinical and radiological evidence of improved tricuspid regurgitation. The Patient was doing well and check echocardiogram done on $13^{\text {th }}$ POD which reveals grade II TR. As he was performing his daily work with minimum difficulty patient was discharged from hospital on $19^{\text {th }}$ POD.

\section{References:}

1. Seo SH, Lee IH, Song YW, Choi BM, Jang GY, Son CS et al. A Case of Congenital Hypertrophic Cardiomyopathy. Korean Circ J. 2013 January; 43: 54-6.

2. Nwaneli CU, Omejua EG, Nwosu NI. Hypertrophic Cardiomyopathy in a Middle Aged Man - A Case Report. Afrimedic Journal 2010; 1:34-7

3. Error! Hyperlink reference not valid., Error! Hyperlink reference not valid., Error! Hyperlink reference not valid.. Secondary tricuspid regurgitation or dilatation: which should be the criteria for surgical repair. Ann Thorac Surg. 2005 Jan;79:127-32.

4. Osterhouse MD, Kettner NW, Boesch R. Congestive Heart Failure: A Review and Case Report From a Chiropractic Teaching Clinic. J Manipulative Physiol Ther 2005 Jun; 28:356-64

5. Sharony R, Grossi EA, Saunders PC. Repair of Tricuspid Regurgitation: The Posterior Annuloplasty Technique. Ann Thorac Surg. 2005 Jan;79:127-32.

6. Jason H. Rogers, MD; Steven F. Bolling, MD. The tricuspid valve, Current Perspective and Evolving Management of Tricuspid Regurgitation. Circulation. 2009; 119: 2718-25. 